\title{
Metamaterials, Metasurfaces, and Artificial Dielectrics: Theory and Applications to the Next-Generation Telecommunication Platforms
}

\author{
Davide Ramaccia, ${ }^{1}$ Ariel Epstein, ${ }^{2}$ Mirko Barbuto, ${ }^{3}$ and Scott Rudolph ${ }^{4}$ \\ ${ }^{1}$ Roma Tre University, Rome, Italy \\ ${ }^{2}$ Technion-Israel Institute of Technology, Haifa, Israel \\ 3 "Niccolò Cusano" University, Rome, Italy \\ ${ }^{4}$ US Naval Research Laboratory, Washington, DC, USA \\ Correspondence should be addressed to Davide Ramaccia; davide.ramaccia@uniroma3.it
}

Received 10 August 2017; Accepted 10 August 2017; Published 11 September 2017

Copyright ( 2017 Davide Ramaccia et al. This is an open access article distributed under the Creative Commons Attribution License, which permits unrestricted use, distribution, and reproduction in any medium, provided the original work is properly cited.

In recent years, wireless telecommunication providers have faced a significant expansion in the number of services demanded by their customers, which requires radiators with increasingly exceptional performance in terms of bandwidth, radiation patterns, space occupancy, and multifunctionality. Simultaneously, metamaterials, metasurfaces, and artificial dielectrics have revolutionized electromagnetism, improving the performance of existing devices and enabling a huge number of new applications.

This special issue is intended to reflect current research and development trends and novel approaches in the application of artificial material, metamaterial, and metasurface for next-generation of telecommunication platforms. A particular emphasis has been paid to components, devices, and systems to be employed in mobile communication towers, satellite payloads, aircraft, radars, and ship masts. Wide-band electrically small metamaterial-inspired antennas, multipleinput and multiple-output antenna systems, multiantenna platforms with invisibility cloaks exhibiting low mutual coupling and reduced blockage between elements, and highly directive low-profile metasurface-based antennas are just some of the novel marvels enabled by this technology.

We are particularly glad to serve as guest editors for this special issue, which contains a representative collection of the current trends and novel applications of artificial material, metamaterial, and metasurface in telecommunication field.
The paper by R. Foster et al. is focused on the beamsteering capabilities of a simplified flat Luneburg lens operating at $60 \mathrm{GHz}$. The lens is designed by using transformation electromagnetics approach and, then, fabricated by using ceramic composites. The beam-steering capabilities of the lens are experimentally verified, demonstrating that it can azimuthally scan a region within $\pm 30^{\circ}$ with gains of at least $18 \mathrm{dBi}$ over a bandwidth from 57 to $66 \mathrm{GHz}$. The lens has been used in a wireless link for the transmission of a raw high definition video.

The paper by E. Öziş et al. presents a review of the literature on microwave radomes for antenna systems made of metamaterials and introduces the concept of "metaradomes." The paper discusses the possibilities that metamaterials open up in the improvement and/or correction of characteristics (gain, directivity, and bandwidth) of the enclosed antenna, as well as the possibility of adding new features, such as bandpass frequency behaviour, polarization transformations, and the ability to be switched on/off. Examples of applications of metamaterials in the design of microwave radomes available in the literature as well as potential applications, advantages, drawbacks, and still-open problems are described.

The paper by G. Artner et al. presents a carbon-fibre reinforced polymer (CFRP) laminate to be used as ground plane material for both a narrowband wire monopole antenna at $5.9 \mathrm{GHz}$ and an ultrawideband conical monopole antenna for 
1-10 GHz. CFRP are already used for lightweight construction of reflectors, mostly in large parabolic dishes or space applications, but they typically present a high anisotropy due to the alignment of shredded fibres. In this paper, the authors design and experimentally verify the possibility of decreasing the material anisotropy by randomly orienting the fibres, which compose the ground plane for both wire and conical monopoles.

The paper by X. Zhao et al. introduces a topological method for the design and optimization of low-profile circularly polarized (CP) directional antenna. The authors propose to insert two layers of artificial materials, generated by particle swarm optimization, between an equiangular spiral antenna and the ground, in order to achieve both directional radiation pattern and high gain. The optimized and manufactured antenna exhibits good impedance matching in the band $4-12 \mathrm{GHz}$, with a whole-band stable directional pattern in 4-11.5 GHz and antenna gain of $8 \mathrm{dBi}$.

L. Su et al. present a review paper on the sensing approaches for the implementation of microwave sensors based on transmission lines loaded with metamaterialinspired resonators. The authors focus the attention on four sensing strategies: (i) resonance frequency variation, (ii) coupling modulation through symmetry disruption (causing variation of the notch depth), (iii) frequency splitting (also exploiting symmetry properties), and (iv) amplitude modulation of a harmonic signal. These sensors are useful in various scenarios, such as characterization of dielectric materials for communication circuits, medical diagnosis and treatment with microwave technologies, and sensors for space applications.

The paper by R. Kubacki et al. presents a microstrip patch antenna based on the left-handed metamaterial concept. By using a planar periodic geometry derived from fractal shapes, the antenna exhibits improved characteristics in terms of bandwidth, gain, and radiation characteristics. The proposed design could support an ultrawide bandwidth ranging from 4.1 to $19.4 \mathrm{GHz}$, demonstrating enhanced gain with an average value of $6 \mathrm{dBi}$ over the entire frequency range, a peak of $10.9 \mathrm{dBi}$, and a radiation capability directed in the horizontal plane of the antenna.

The paper by J. Gao et al. presents a different parameter extraction approach based on zero immittances for composite right/left-handed (CRLH) structures. For lossless unit cell equivalent circuit model, L-C parameters of series and parallel branches are extracted from the series and parallel resonance frequencies, respectively. This approach, which can be applied to symmetric and unbalanced CRLH structures, provides an effective and straightforward way to design CRLH metamaterials for applications in telecommunication systems.

\section{Acknowledgments}

We would like to acknowledge the many people that have made the publication of this special issue possible. First of all, sincere thanks go to the authors for having submitted the exciting results of their research to this special issue. We are also very grateful to the reviewers who dedicated their time and expertise to increase the scientific quality of the submissions. Finally, the guest editors would sincerely like to thank all the readers interested in the papers published on this special issue. We hope you may find here new exciting ideas for your research activities.

Davide Ramaccia Ariel Epstein Mirko Barbuto Scott Rudolph 


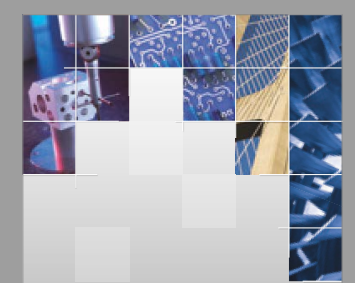

\section{Enfincering}
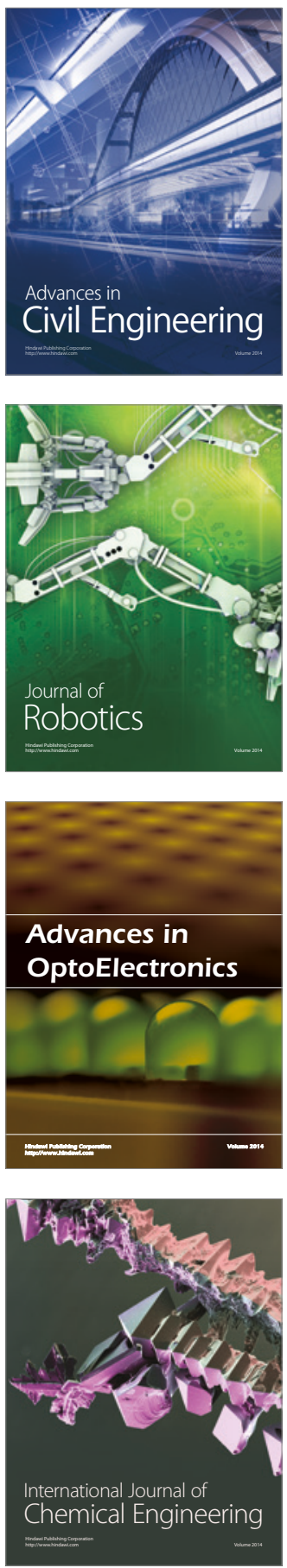

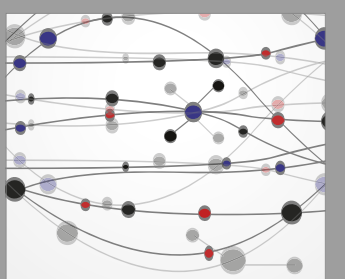

The Scientific World Journal

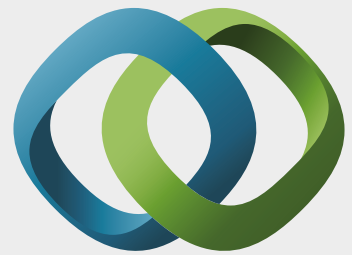

\section{Hindawi}

Submit your manuscripts at

https://www.hindawi.com
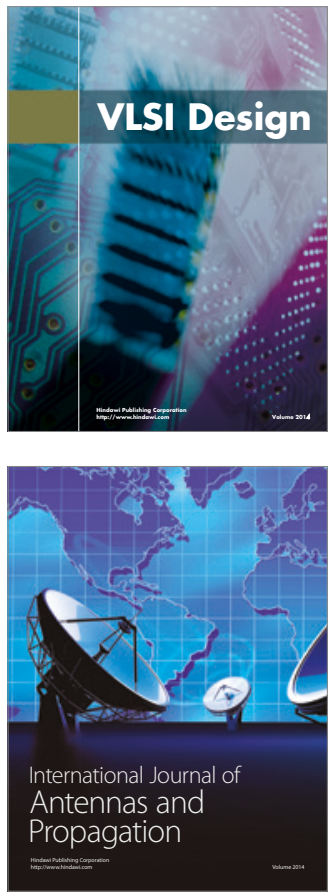

\section{Rotating}

Machinery
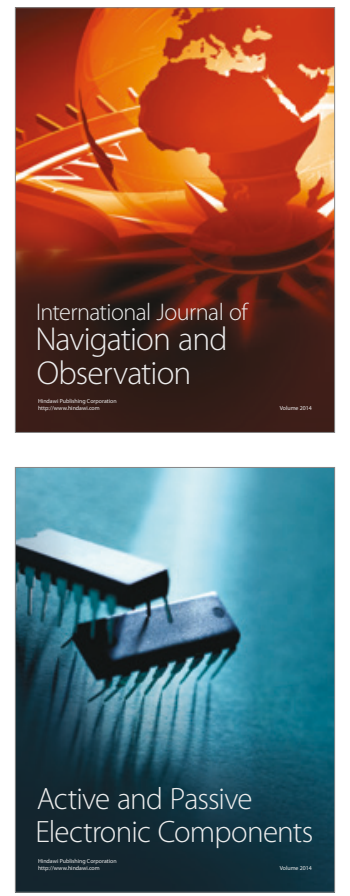
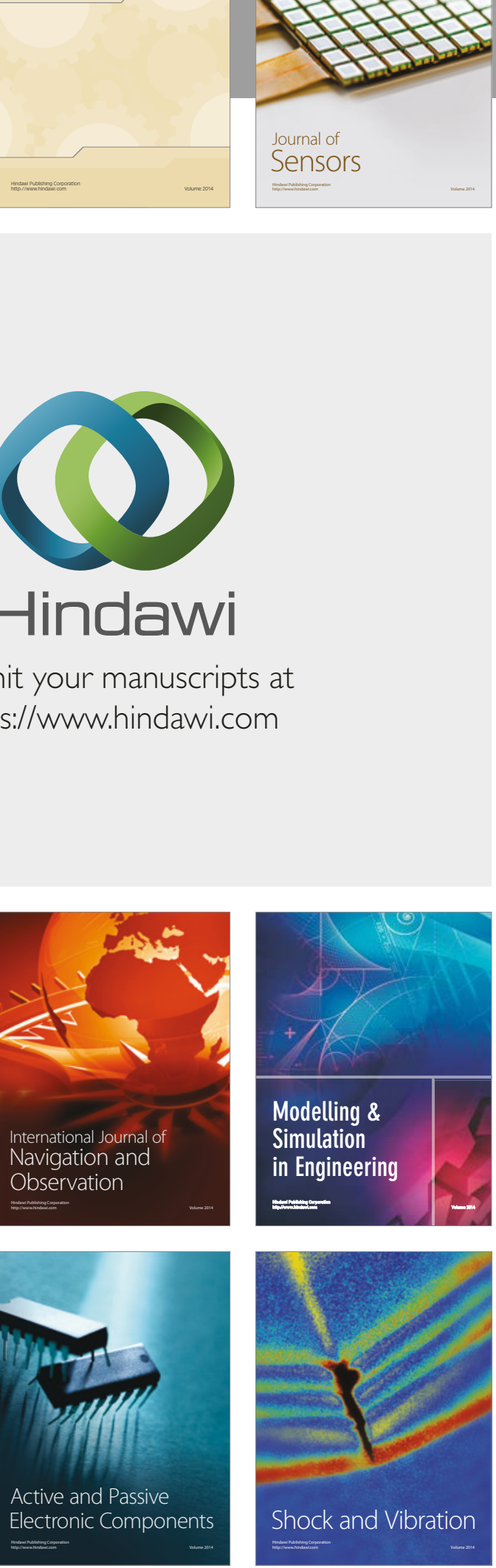
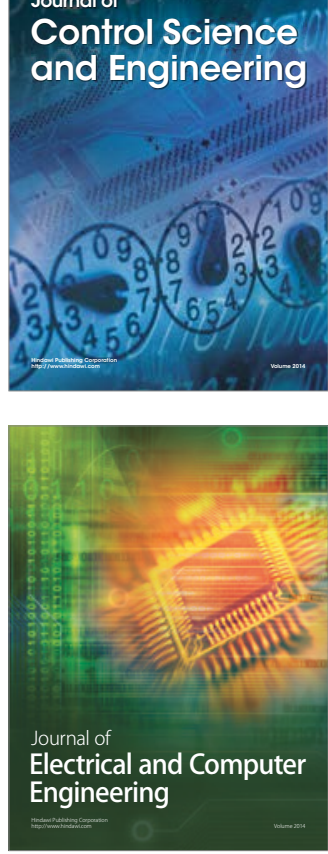

Distributed

Journal of

Control Science

and Engineering
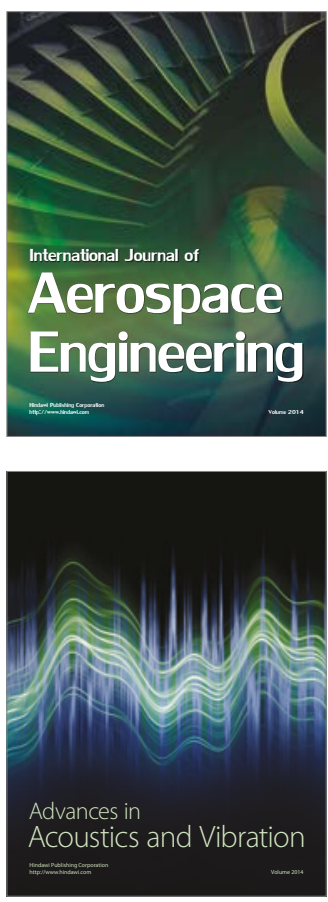

Sensor Networks 